\title{
Stress Behaviour Improvement of Automobile Flywheel Under High Centrifugal Forces
}

\author{
Çağlar İmer, Samet Kartal, Mehmet Onur Genç \\ Valeo Automotive \\ Bursa, Turkey \\ caglar.imer@valeo.com; samet.kartal@valeo.com; mehmet-onur.genc@valeo.com
}

\begin{abstract}
Clutch components rotating at high speed remain under the influence of high centrifugal forces. Therefore at the high rotational speed flywheel is subjected to extreme forces that may cause severe cracks and breakages. During driving condition vehicle can be used in different engine RPM. Burst test validates the mechanical robustness of the flywheel under various rotational speeds. In this study finite element analysis has been used for calculating stress values of flywheel. In order to simulate burst test, finite element method can be used that provides estimation instead of prototypes production which causes time and money consumption.

Additionally design of experiment method has been used for obtaining the response surface that approximates the stress behaviour of automobile flywheel. Data fitting method was applied to determine objective function. Two shape parameters have been selected for observing change of stress value. It provides strong correlation between fea results and data fittings calculation that gives extra contribution to reduce the design process.
\end{abstract}

Keywords: centrifugal forces, flywheel, experiment method, stress replacement, response surface

\section{Introduction}

Flywheel is one of the main component of the transmission system in terms of safety. The flywheel is directly exposed to high centrifugal forces. Due to the importance of the flywheel, the durability of the flywheel must be investigated under high centrifugal force.

In this study, flywheel verification process is described with simulation results. As a result of the study, developed finite element model is matched well with the experimental results.

Charles (2012) investigate in the study mechanical properties of flywheel in terms of energy storage and mass regulator component. Flywheel is the energy storage component that keeps the dissipated energy with the high inertia and material characteristic. This study includes the experimental and mathematical approach to powertrain system analysis. In order to make further analysis flywheel constraints are defined coming from rotational forces and heat transfer [1]. Abdullah et al. (2013) studied the frictional clutch components relations in terms of stress and deformations. In the study contact points during driving condition were investigated by using finite element method. Prominent conclusion is clutch system is subjected to dynamic forces during high rotational speed and contact points between clutch cover and flywheel should be optimized to reduce stress that cause crack and breakage [2].

Maninder et al. (2015) studied in this paper the optimization of flywheel mass by using computer aided analysis. Flywheel optimized geometry was investigated by using specific energy calculation in terms of components life limit. Authors emphasized in the study that flywheel geometry, mass and stress distribution have strong relation with each constraint [3]. In this study automotive flywheel is subjected to FEA analysis to investigate burst improvement by response surface methodology. This paper approaches to stress improvement to vehicle flywheel both FEA and optimization methodology. 


\section{Material and Method}

\subsection{FEA Study}

In this study, the components in vehicles subjected to high rotational speed are investigated in terms of the stress behavior on design variations with FEA analysis for real conditions. In order to validate the design of flywheel under high speed condition, burst test is performed with adapted bench test which simulates as in vehicle. Duration of preparing of test parts takes longer times due to prototyping processes such as casting mold production and finding available schedule in serial production lines of automotive industry plants. Also implementation of flywheel prototypes with different designs is cost due to produce a casting mold for each flywheel design. Therefore finite element solutions are helpful to improve the flywheel design in short time.

Flywheel design target is to withstand at $12000 \mathrm{rpm}$ to simulate the worst condition in vehicle. Experimental data presents that maximum stress area located near the clutch cover fixation holes due to severe section transitions. Also it is known that flywheel is safety component for powertrain systems and on the study stress behaviours near the critical location improvement was aimed. At the beginning of the study, flywheel was designed without slots in figure 1 . Slot geometry was considered to replace the maximum stress from critical area to another non-functional area. Previous design was gathering the stress into severe sections such as holes.

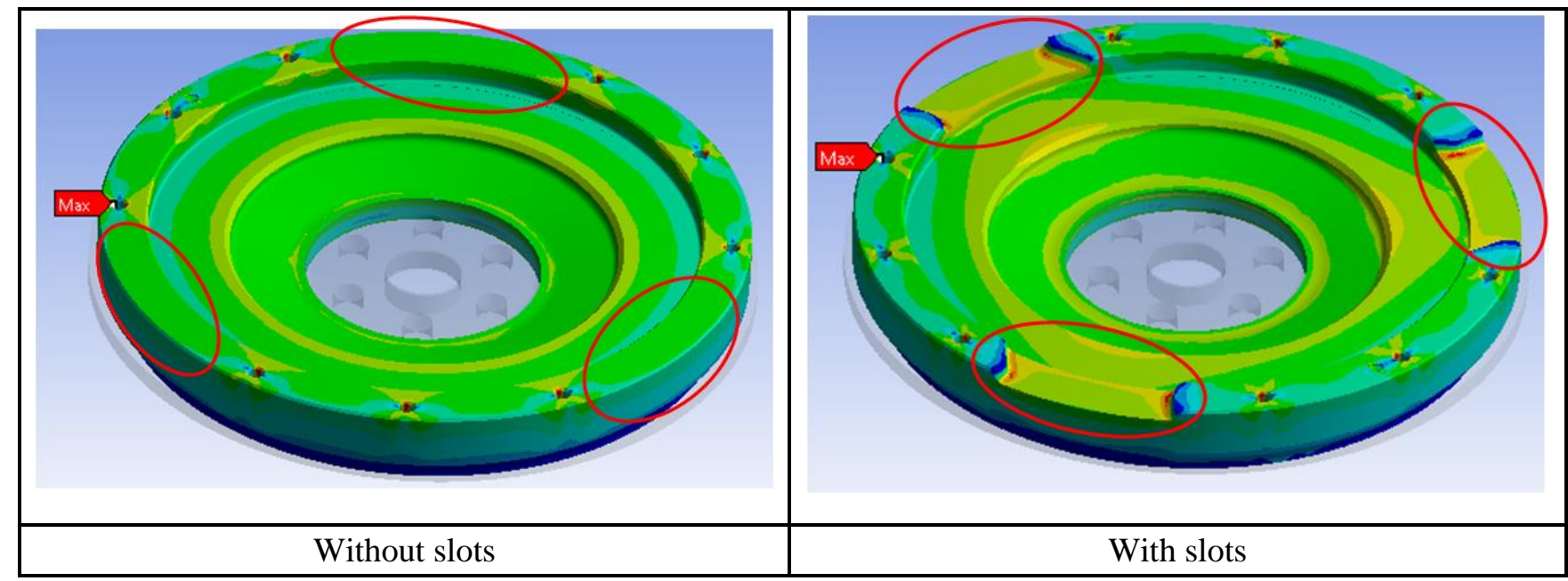

Fig. 1: Design creation with slots.

In the finite element model, there are three components following flywheel, ring gear and a fake crankshaft. Fake crankshaft was fixed from engine side. As in shown figure 2, two contact areas were defined in simulation model. First contact is between crankshaft and flywheel. Contact type was chosen as frictional. Second contact is between ring gear and flywheel due to shrink fitting. This contact type was chosen as frictional.

In terms of cost and machining, laminar cast iron is practical solution for flywheels. Therefore cast iron material was chosen as GJL300 laminar cast iron. Ring gear material was chosen C40 steel material. If we compare the tensile strength of ring gear and flywheel material, ring gear material have higher tensile strength than flywheel material. Maximum vonmises stress was investigated on flywheel with the guide of this information.

Centrifugal forces effect mostly areas which are located at severe section changes and outer diameters. Therefore fixation holes should be well meshed to calculate stress level which closed to real conditions. At the mesh model, outer shapes were meshed with tetrahedron elements as shown in figure 3. Less critical areas were meshed with quad elements to decrease the element numbers of model. Thus, calculation time was minimized for each design variation. 


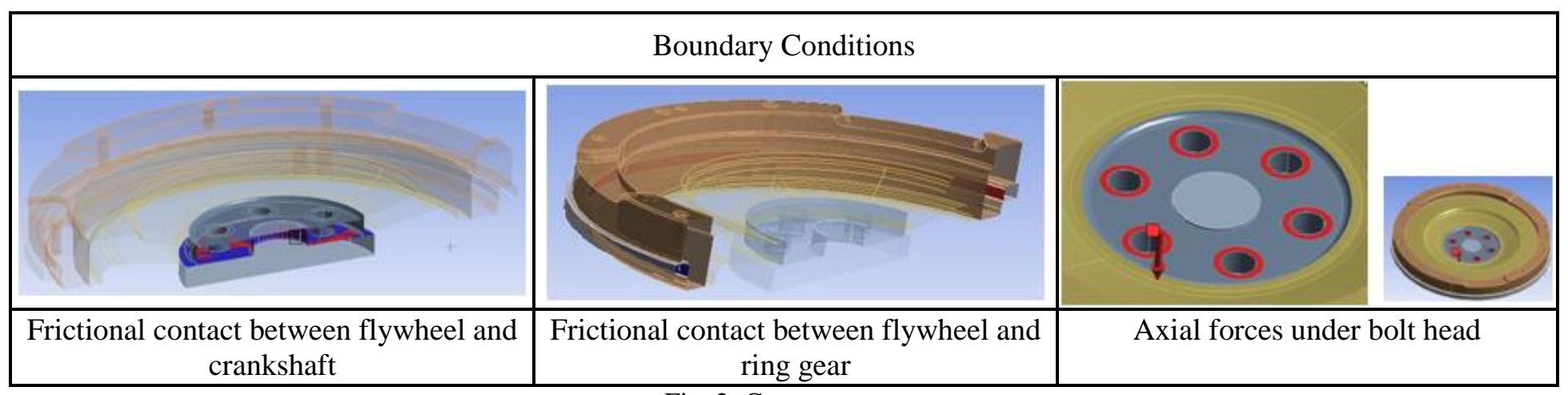

Fig. 2: Contact types.

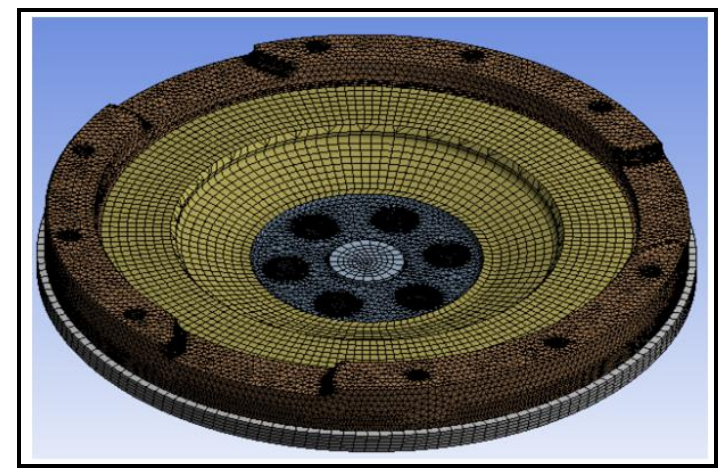

Fig. 3: Mesh model.

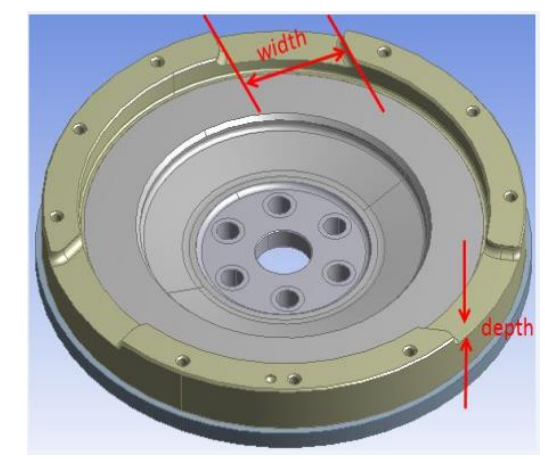

Fig. 4: Design parameters (Slot width and slot depth).

\subsection{Data Fitting}

Flywheel burst simulations which give stress value induced of flywheel at maximum engine speed is necessary to verify flywheel design. A mathematical model analysis run is executed about five hours for flywheel burst simulation. Therefore in this study, response surface methodology is used for obtaining objective function of flywheel stress behaviour. In order to obtain objective function firstly geometrical model of flywheel was created. General shape of flywheel was designed according to limitations of clutch house space. With slot geometry subtraction flywheel design was finalized. Slot geometry subtraction was built for stress replacement as cyclic symmetric at $120^{\circ}$. Two shape parameters were determined for slot geometry that effects to stress replacement directly.

Then a mathematical model was created that best fits to the data which collected from experimental flywheel burst test results. According to full factorial design with four level and two parameters as shown in figure $4,2^{4}=16$ finite element analyses were executed for define a surface that approximates the behaviour of the objective function. According to design of experiment results, objective function was constructed as second degree polynomial. Objective function;

\section{ICMIE 120-3}




$$
f=224.687+4.135 x X+(4754.647 / Y)+\left(-97781.663 /\left(Y^{2}\right)\right)
$$

\section{Analysis and Discussions}

After model creation, sixteen fea study were implemented to find von-mises stress level against to change of slot width and depth. As shown on figure 5, most critical areas are similar locations at outer diameter and on fixation holes as mentioned as described in section 2.1. The both parameters especially were selected applicable values according to design constraints. Calculated results at $12000 \mathrm{rpm}$ with simulations create an indicator database to find the relationship between two design parameters and maximum von-mises stress. As showed in table 1, sixteen fea results are listed according to two design parameters.
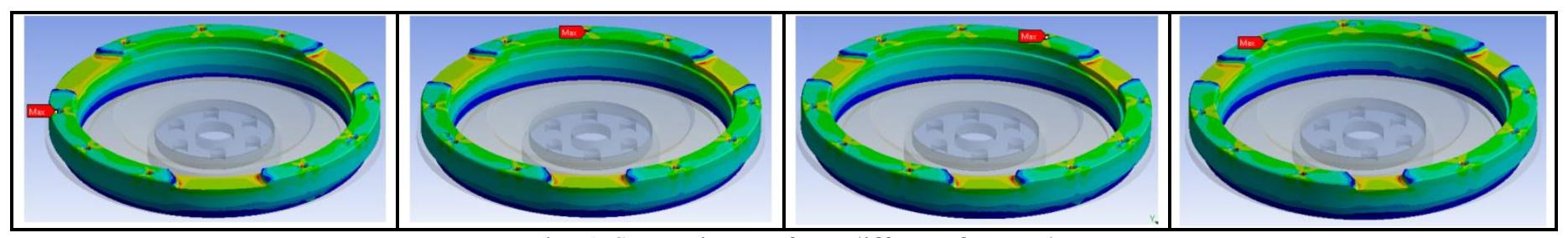

Fig. 5: Some pictures from different fea results.

If listed results are filtered, it is seen that slot design helped to decrease the stress level from situation of initial flywheel design. Maximum stressed area was relieved thanks to another severe section changes on flywheel. The best improvement was seen with design number 1 includes depth $5 \mathrm{~mm}$ and width $68 \mathrm{~mm}$ parameters. In initial design, maximum stressed area was $318.42 \mathrm{MPa}$ on fixation hole at outer diameter. Thus, there was a stress decrease of $7.6 \%$ at maximum stressed area from 318.42 MPa to 294.07 MPa. But some design parameters could not improve the stress level. Design number 15 includes $8 \mathrm{~mm}$ slot depth and $48 \mathrm{~mm}$ slot width. This parameter combination cannot give an improvement due to that it has similar results about $318 \mathrm{MPa}$ as in initial design.

Table 1: Fea results and objective function results at $12000 \mathrm{rpm}$.

\begin{tabular}{|c|c|c|c|c|c|}
\hline & Slot Depth (X) & Slot Width (Y) & $\begin{array}{c}\text { Objective Function } \\
\text { Result }\end{array}$ & Fea Result & $\begin{array}{c}\text { Deviation between Fea Result and } \\
\text { Objective Function }\end{array}$ \\
\hline Initial Design & Not available & Not available & Not available & 318.42 & Not available. \\
\hline Design 1 & 5 & 68 & 294,136726 & 294,07 & $-0,02 \%$ \\
\hline Design 2 & 5 & 58 & 298,2715907 & 298,12 & $-0,05 \%$ \\
\hline Design 3 & 5 & 48 & 301,9771879 & 301,3 & $-0,22 \%$ \\
\hline Design 4 & 5 & 38 & 302,7684564 & 305,43 & $0,88 \%$ \\
\hline Design 5 & 6 & 68 & 298,271726 & 295,42 & $-0,96 \%$ \\
\hline Design 6 & 6 & 58 & 302,4065907 & 299,72 & $-0,89 \%$ \\
\hline Design 7 & 6 & 48 & 306,1121879 & 301,22 & $-1,60 \%$ \\
\hline Design 8 & 6 & 38 & 306,9034564 & 305,33 & $-0,51 \%$ \\
\hline Design 9 & 7 & 68 & 302,406726 & 301,78 & $-0,21 \%$ \\
\hline Design 10 & 7 & 58 & 306,5415907 & 308,21 & $0,54 \%$ \\
\hline Design 11 & 7 & 48 & 310,2471879 & 310,65 & $0,13 \%$ \\
\hline Design 12 & 7 & 38 & 311,0384564 & 312,83 & $0,58 \%$ \\
\hline Design 13 & 8 & 68 & 306,541726 & 309,47 & $0,96 \%$ \\
\hline Design 14 & 8 & 58 & 310,6765907 & 314,79 & $1,32 \%$ \\
\hline Design 15 & 8 & 48 & 314,3821879 & 318,67 & $1,36 \%$ \\
\hline Design 16 & 8 & 38 & 315,1734564 & 322,49 & $2,32 \%$ \\
\hline
\end{tabular}

ICMIE 120-4 
Additionally, objective function results were listed on table 1. Used parameters in fea calculations re-calculated with objective function equations. The both calculations of objective function and fea result were compared to show the deviation ratio. Comparing to simulated parameters in finite element models, objective function have maximum $2.32 \%$ deviation in terms of stress level. If it is considered with calculation time of 16 finite element models, they take approximately 80 hours totally. Whereas objective function can easily calculate the results in only a few seconds by any parameters which in range of maximum and minimum design constraints.

According to objective function results, response surface is shown on figure 6 . When the response surface is analyzed, it is observed that the results do not change with linear trend. In terms of better stress level, slot width should be wider and slot depth should be lesser depth in this type of flywheel which has similar design. With response surface method, best parameter combination can be chosen on graph. In this way, a fast prediction can be performed in the beginning of the project.

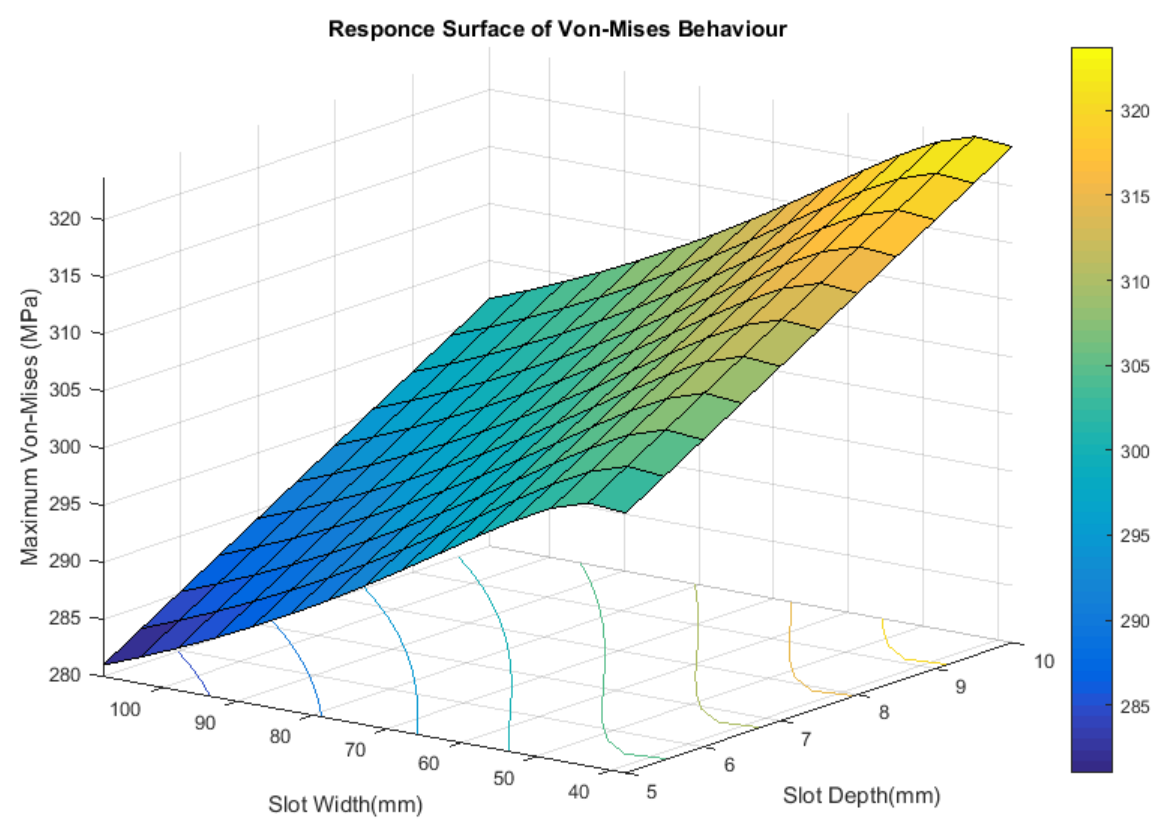

Fig. 6: Response surface graph of objective function.

\section{Conclusion}

Today, new engine technology requirements are high speed, lower volume and safety become important in automotive industry. These constraints make hard to create robust flywheel design. Flywheel is not only torque transfer component also robust design of flywheel has importance due to safety of driver and passengers. Therefore improvement of stress level on flywheel is important design progress.

After design change with slots creation, stress improvement was observed. The study shows that slot design helps to replace the maximum stress from most critical area to non-functional area. Sixteen different design studies were realized with fea calculations to find the experimental values. With design of experiment study, flywheel development process has been completed faster and cheaper. It will be used an optimization method for robust and efficient flywheel design process on next time studies.

\section{References}

[1] X. Charles, "Design and Stress Analysis of Composite Material Flywheel in Automotive," Middle-East Journal of Scientific Research, vol. 12, no. 12, pp. 1620-1624, 2012.

[2] O. I. Abdullah, J. Schlattmann, A. M. Al Shabibi, "Stresses and Deformations Analysis of a Dry Friction Clutch System," Tribology in Industry, vol. 35, no. 2, pp. 155-162, 2013.

[3] S. Bhardwaj Maninder, "Design and Analysis of Light Weight Motor Vehicle Flywheel," International Journal 
of Innovation Sciences and Research, vol. 4, no. 8, pp. 361-365, 2015.

[4] S. Gupta, K. Kothari, J. P. Mishra, B. Ashok J., "Optimization of Gasoline Engine Flywheel using Alternate Materials for Improving Performance Characteristics," Indian Journal of Science and Technology, vol. 8, no. 30, DOI:10.17485/ijst/2015/v8i30/72443, 2015.

[5] O. I. Abdullah, J. Schlattmann, "Contact Analysis of a Dry Friction Clutch System," Hindawi Publishing Corporation, ISRN Mechanical Engineering, Article ID 495918, 2013.

[6] R. Purohita, P. Khitoliyab, D. K. Kolic, 'Design and Finite Element Analysis of an Automotive Clutch Assembly" 3rd International Conference on Materials Processing and Characterisation, Procedia Materials Science, vol. 6, pp. 490-502, 2014.

[7] M. Lavakumar, R. P. Srınıvas, "Design and Analysis of Light Weight Motor Vehicle Flywheel," International Journal of Computer Trends and Technology (IJCTT), vol. 4, no. 7, 2013.

[8] M. K. Tipirineni, P. P. Rao, "Optimal Design of a Clutch Plate using Ansys," International Journal of Computational Engineering Research, vol. 03, no. 12, 2013. 